\title{
Época de nascimento, genótipo e sexo de terneiros cruzas taurinos e zebuínos sobre o peso ao nascer, à desmama e eficiência individual de primíparas Hereford ${ }^{1}$
}

\author{
Calving season, genotype and sex of crossbred taurus and indicus calves on the birth \\ and weaning weight and individual efficiency of primiparous Hereford
}

\author{
Gilson de Mendonça ${ }^{2}$ Marcelo Alves Pimentel ${ }^{3}$ Ricardo \\ Alberto Cardellino $^{3}$ José Carlos da Silveira Osório $^{3}$
}

RESUMO

\begin{abstract}
O objetivo deste trabalho foi avaliar o efeito da época de nascimento, genótipo e sexo do terneiro sobre a eficiência individual das vacas à desmama (relação percentual entre o peso do terneiro à desmama e o peso da vaca), peso ao nascer e peso à desmama dos terneiros. Foram utilizadas 48 vacas da raça Hereford (Bos taurus), com idade de três anos, manejadas sobre campo natural, 16 inseminadas com um touro da raça Red Angus (Bos taurus) e $32 \mathrm{com}$ Nelore (Bos indicus). Os fatores estudados foram genótipo do terneiro (GENOT) - Bos taurus $x$ Bos taurus e Bos taurus $x$ Bos indicus, época de nascimento (EPOCN) subdividida em três períodos - $1 \mathrm{~S}$ (setembro), 20 (outubro) e $3 N$ (novembro) e sexo do terneiro (SEXO). A produção de leite (PL) foi incluída como co-variável. Foram realizadas dez medidas de produção de leite pelo método indireto pesagem - mamada - pesagem e onze medidas de peso para vacas e terneiros, em intervalos de 21 dias. Os fatores que influenciaram significativamente o PN foram GENOT e SEXO $(P<0,05)$. O P205 sofreu influência significativa da EPOCN, SEXO e PL $(P<0,05)$. A EI foi influenciada significativamente pela EPOCN, SEXO e PL até 147 dias $(P<0,05)$. Concluiu-se que genótipo e sexo dos terneiros influenciam o peso ao nascer. $O$ peso à desmama e a eficiência das vacas são influenciados pelo sexo e época de nascimento, mas não pelo genótipo dos terneiros.
\end{abstract}

Palavras-chave: bovinos de corte, eficiência da vaca, peso ao desmame, peso ao nascer.

\section{ABSTRACT}

The objective of the present study was to evaluate the effect of calving season, genotype and calf sex on the cows

\begin{abstract}
weaning individual efficiency ( percentual relationship between cow body weight and calf weaning weight), birth and weaning weight of calves. Forty-eight primiparous Hereford cows with approximate average age of 3 years and grazing only native pastures were used. Sixteen inseminated with one Red Angus bull (Bos taurus) and 32 with Nelore (Bos indicus). Effects studied were calf genotype (GENOT), Bos taurus $x$ Bos taurus and Bos taurus $x$ Bos indicus, calving season (EPOCN) concentrated in the Spring and subdivided into three periods: $1 S$ (September), $2 O$ (October) and $3 N$ (November and December) and calf sex (SEXO). Milk production (PL) was included as co-variable. Ten measurements of milk production obtained by the indirect method, weighing the calf before and after suckling, and eleven measurements of cow and calf weights, were made at 21 day intervals. Effects with significant influence on PN were GENOT and SEXO $(P<0.05)$. P205 was significantly affected by EPOCN, SEXO and PL (P<0.05). EI was significantly affected by EPOCN, SEXO and PL until 147 days $(P<0.05)$. Calf genotype and sex affected their birth weight. Weaning weight and cow efficiency are affected by sex and and calving season, but not by calf genotype.
\end{abstract}

Key words: beef cattle, cow efficiency, birth weight, weaning weight.

\section{INTRODUÇÃO}

Os índices de produtividade dos rebanhos de corte brasileiros têm demonstrado pouco incremento nos últimos dez anos e sua melhoria é condição indispensável para a manutenção da atividade. A determinação da eficiência individual das

${ }^{1}$ Parte da Dissertação do primeiro autor para obtenção do grau de Mestre em Zootecnia

${ }^{2}$ Professor, Departamento de Zootecnia, Faculdade de Agronomia Eliseu Maciel (FAEM), Universidade Federal de Pelotas (UFPEL), Campus Universitário s/n, CP 354, 96010-900, Pelotas, RS. Email:mendonca@ufpel.tche.br Autor para correspondência.

${ }^{3}$ Professor, Departamento de Zootecnia, FAEM-UFPEL, Campus Universitário s/n, CP 354, 96010-900 Pelotas, RS. Email:map@ufpel.tche.br 
vacas à desmama (relação percentual entre o peso do terneiro e da vaca) é uma ferramenta importante para isto, uma vez que reflete as vantagens de um determinado tipo ou tamanho de gado e raça em um determinado ambiente. A identificação de sistemas de acasalamento adequados e de épocas diferenciadas de acasalamento/parição são alternativas para o aumento da eficiência, e dentre os vários fatores que podem influenciá-la estão o sexo, o peso ao nascer, o peso à desmama e o genótipo dos terneiros (LOBATO, 1984; LOBATO et al., 1999).

A época de nascimento é um dos principais fatores que influenciam o peso ao nascer dos terneiros. Vacas com parição na primavera têm, no terço final da gestação, disponibilidade alimentar insuficiente para atender à demanda nutricional do máximo crescimento fetal, produzindo terneiros mais leves ao nascimento. Por outro lado, essas mesmas vacas contam com melhor nível nutricional no terço final da lactação, proporcionando ganho médio diário e peso à desmama maior aos seus filhos (BARCELLOS \& LOBATO, 1992a; 1992b). Geralmente, o peso ao nascer aumenta à medida que avança a época de nascimentos (ROVIRA, 1974; FRANZO, 1997). De acordo com ALENCAR (1987), terneiros, quando muito jovens, dependem exclusivamente do leite materno, sendo os efeitos do dia de nascimento não muito importantes, tornando-se significativos somente quando passarem a se alimentar do pasto disponível.

Outro fator que pode influenciar o peso ao nascer, assim como o peso à desmama e, conseqüentemente, a eficiência das vacas, é o genótipo dos terneiros. Terneiros oriundos do cruzamento Bos taurus $\mathrm{x}$ Bos indicus manifestam sob efeito da heterose maior vigor geral, maior peso ao nascer que a média das raças incluídas no cruzamento e maior ganho de peso (KOGER et al., 1976; CARDELLINO \& ROVIRA, 1987; BARCELLOS \& LOBATO, 1992b).

Com relação ao sexo, naturalmente, terneiros machos são maiores e mais pesados do que fêmeas ao nascimento, mantendo-se essa diferença à desmama (ROVIRA, 1974; JARDIM \& PIMENTEL, 1998).

Estudos têm verificado associação significativa entre a produção de leite e o ganho de peso dos terneiros, sendo a mesma responsável por uma porção significativa da variação nos pesos dos terneiros à desmama e nos ganhos de peso do nascimento à desmama (BOGGS et al., 1980; ALENCAR et al., 1985; MALLINCKRODT et al., 1993; ALENCAR et al., 1996; ROVIRA, 1996). ALBUQUERQUE et al. (1993) encontraram correlação fenotípica entre produção de leite e peso à desmama dos terneiros de 0,71 , evidenciando forte associação entre essas duas características.

O objetivo deste trabalho foi avaliar o efeito da época de nascimento, genótipo e sexo do terneiro sobre a eficiência individual das vacas à desmama, peso ao nascer e peso à desmama dos terneiros, assim como relacionar a produção de leite com essas variáveis.

\section{MATERIAL E MÉTODOS}

O trabalho foi conduzido numa fazenda situada no município de Aceguá, RS. A região apresenta clima mesotérmico, tipo subtropical da classe Cfa 1, e a distribuição das chuvas durante o ano é $34 \%$ no inverno, $25 \%$ na primavera, $16 \%$ no verão e $25 \%$ no outono. Ocasionalmente, entre os meses de novembro a março, podem ocorrer períodos secos (MACEDO, 1984). A temperatura média anual é de $17,6^{\circ} \mathrm{C}$, sendo janeiro o mês mais quente e junho o mais frio. Há formação de geadas entre os meses de abril a novembro, com maior incidência de junho a agosto. A umidade relativa do ar oscila entre 75 e $85 \%$. O relevo da região na qual está localizado Aceguá, é suavemente ondulado, apresentando longos declives de 2 a $8 \%$, chegando em determinadas áreas a possuir topografia plana. A fertilidade natural do solo é moderada, principalmente pelos baixos teores de fósforo. A cobertura natural do solo é superior a $80 \%$, sendo formada por vegetação nativa com predominância de gramíneas de ciclo estival, principalmente grama forquilha (Paspalum notatum) e grama tapete (Axonopus affinis), apresentando variações qualitativas nos diferentes períodos do ano. Nos períodos de primavera e verão, são baixos e densos, formando uma cobertura natural de boa qualidade para exploração sob pastagem. No período de inverno (junho a agosto), não apresentam crescimento e ficam secos pela ocorrência de geadas.

Foram utilizadas 48 vacas da raça Hereford, primíparas, com idade de três anos, manejadas sobre campo natural. A lotação utilizada foi $0,7 \mathrm{UA} \cdot \mathrm{ha}^{-1}(1 \mathrm{UA}=450 \mathrm{~kg}$ de peso vivo $)$. As raças paternas utilizadas, por meio de inseminação artificial, foram Red Angus e Nelore (1 reprodutor de cada raça), em 16 e 32 vacas respectivamente. A época de parição concentrou-se na primavera, nos meses de setembro, outubro e novembro. O desmame foi realizado quando os animais apresentavam em média 210 dias de idade. A época de parição foi subdividida em três períodos: $1 \mathrm{~S}$ (nascimentos em setembro), $2 \mathrm{O}$ (nascimentos em outubro) e $3 \mathrm{~N}$ (nascimentos em novembro). 
Foi realizada a medição da produção de leite $(\mathrm{kg})$ por meio do método utilizado por GREEN et al. (1991). As medições foram realizadas em intervalos de 21 dias, à partir da média da data de nascimento, num total de 10 medidas, totalizando-se dessa maneira um período de lactação de 210 dias. Juntamente foram realizadas medições do peso dos animais. Foi utilizada uma balança eletrônica da marca Tru-Test, com capacidade máxima de $1500 \mathrm{~kg}$ e precisão de $100 \mathrm{~g}$.

Foram analisadas três variáveis resposta: peso ao nascer dos terneiros $(\mathrm{PN}), \mathrm{em} \mathrm{kg}$; peso à desmama ajustado para 205 dias (P205), em kg e eficiência individual das vacas à desmama (EI), em percentual. Os cálculos da EI e P205 foram obtidos pela fórmula utilizada pelo PROMEBO (ANC HERD BOOK COLLARES, 1976). As médias foram comparadas pelo teste de Tukey $(\mathrm{P}<0,05)$. Na análise, utilizou-se o PROC GLM do Statistical Analysis System (SAS, 2000).

Foram analisados três fatores que podem influenciar o PN, P205 e a EI, assim como a interação entre eles: época do nascimento (EPOCN), com três níveis - $1 \mathrm{~S}$ (setembro), $2 \mathrm{O}$ (outubro) e $3 \mathrm{~N}$ (novembro); genótipo do terneiro (GENOT), com dois níveis $-\mathrm{N}$ (Nelore) e RA (Red Angus); e sexo do terneiro (SEXO), com dois níveis - F (fêmea) e M (macho).

Para efeito de análise, foram incluídas como co-variáveis as três principais fases da curva de lactação, sendo o aumento de produção até o pico ( $\left.\mathrm{PL}_{\mathrm{P} 84}\right)$, o declínio ( $\left.\mathrm{PL}_{\mathrm{P} 147}\right)$ e a estabilização final ( $\left.\mathrm{PL}_{\mathrm{P} 210}\right)$, produções de leite acumuladas até 84, 147 e 210 dias de lactação, respectivamente. As produções de leite, nos períodos, foram estimadas pela fórmula proposta por ALENCAR et al. (1996).

\section{RESULTADOS E DISCUSSÃO}

A média geral de $\mathrm{PN}$ foi $30,41 \pm 0,66 \mathrm{~kg}$, sendo inferior à obtida por FRANZO (1997) e RIBEIRO \& LOBATO (1988). A EPOCN não teve influência significativa sobre o $\mathrm{PN}(\mathrm{P}>0,05)$, resultado este que não concorda com os obtidos por ROVIRA (1974) e FRANZO (1997) segundo os quais o peso dos terneiros ao nascer aumentou de acordo com o avanço da época de nascimentos. No presente trabalho, os resultados encontrados parecem indicar que não houve oferta forrageira suficiente para atender a maior demanda de nutrientes das vacas no final de gestação, à medida que avançava a época de nascimento, conseqüentemente, não houve incremento no peso ao nascer dos terneiros. Por outro lado, a EPOCN influenciou de maneira significativa o P205 $(\mathrm{P}<0,05)$, sendo que os terneiros nascidos no período $1 \mathrm{~S}$ apresentaram maiores pesos em comparação com os demais. Este resultado está de acordo com o obtido por ALENCAR (1987) segundo o qual o dia de nascimento apresentou efeito linear e quadrático sobre os pesos aos 120 e 240 dias de idade. Da mesma forma, a EI foi influenciada pela EPOCN $(\mathrm{P}<0,05)$, sendo que as vacas paridas no período $1 \mathrm{~S}$ apresentaram maior eficiência comparadas com as demais. Como o peso à desmama é um dos elementos incluídos no cálculo da EI, fica visível a relação existente entre EPOCN e EI. As médias de PN, P205 e EI de acordo com a EPOCN podem ser observadas na tabela 1 .

O GENOT influenciou significativamente $(\mathrm{P}<0,05)$ o $\mathrm{PN}$, observando-se média maior para os filhos de Nelore em comparação aos filhos de Red Angus (Tabela 2). Esses resultados estão de acordo com os encontrados por BARCELLOS \& LOBATO (1992a), que encontraram uma média de peso ao nascer maior para terneiros oriundos do cruzamento de Nelore com vacas Hereford, comparados com a raça pura. Por outro lado, o GENOT não influenciou o $\mathrm{P}_{2} 0_{5}(\mathrm{P}>0,05)$, resultado esse diferente do relatado por BARCELLOS \& LOBATO (1992b), em que o genótipo do terneiro foi

Tabela 1 - Médias de peso ao nascer $(\mathrm{PN})$, peso à desmama ajustado para 205 dias (P205) e eficiência individual das vacas (EI) de acordo com a época de nascimento $(\mathrm{EPOCN})$.

\begin{tabular}{lccc}
\hline & \multicolumn{3}{c}{ EPOCN } \\
\cline { 2 - 4 } Variáveis & $1 \mathrm{~S}$ (setembro) & $2 \mathrm{O}$ (outubro) & $3 \mathrm{~N}$ (novembro) \\
\cline { 2 - 4 } & $30,76 \pm 1,16 \mathrm{a}$ & $29,88 \pm 1,04 \mathrm{a}$ & $31,07 \pm 1,64 \mathrm{a}$ \\
PN & $151,54 \pm 4,86 \mathrm{a}$ & $147,12 \pm 3,96 \mathrm{ab}$ & $143,47 \pm 5,05 \mathrm{~b}$ \\
P205 & $46,89 \pm 2,00 \mathrm{a}$ & $42,75 \pm 1,15 \mathrm{ab}$ & $37,11 \pm 1,02 \mathrm{~b}$ \\
EI & &
\end{tabular}

Médias na mesma linha seguidas de letras minúsculas diferentes, diferem significativamente $(\mathrm{P}<0,05)$.

Tabela 2 - Médias de peso ao nascer (PN), peso à desmama ajustado para 205 dias (P205) e eficiência individual das vacas (EI) de acordo com o genótipo (GENOT) e sexo (SEXO) do terneiro.

\begin{tabular}{lcc}
\hline \multirow{2}{*}{ Variáveis } & \multicolumn{2}{c}{ GENOT } \\
\cline { 2 - 3 } & $\mathrm{N}($ Nelore) & RA (Red Angus) \\
\hline PN & $31,69 \pm 0,85 \mathrm{a}$ & $28,12 \pm 1,13 \mathrm{~b}$ \\
P205 & $147,60 \pm 3,18 \mathrm{a}$ & $148,18 \pm 4,88 \mathrm{a}$ \\
EI & $42,46 \pm 1,37 \mathrm{a}$ & $43,47 \pm 1,60 \mathrm{a}$ \\
\hline
\end{tabular}

Médias na mesma linha seguidas de letras minúsculas diferentes, diferem significativamente $(\mathrm{P}<0,05)$.

Ciência Rural, v. 33, n. 6, nov-dez, 2003. 
o fator que mais afetou o desempenho aos 205 dias, com os terneiros oriundos do cruzamento Bos taurus $\mathrm{x}$ Bos indicus (Nelore x Hereford) apresentando média de peso $16,3 \%$ superior aos originados pelo cruzamento Bos taurus x Bos taurus (Hereford x Hereford).

O GENOT não influenciou a EI $(\mathrm{P}>0,05)$, o que pode ser devido à ausência de efeito do mesmo sobre o P205, como já foi descrito anteriormente. Nesse caso, a restrição alimentar pode ter sido fator limitante para a manifestação de diferenças entre os fatores.

$\mathrm{O}$ PN dos terneiros sofreu influência significativa $(\mathrm{P}<0,05)$ do SEXO, verificando-se uma média maior para machos. Resultados semelhantes foram encontrados por NEWMAN \& DELAND (1991), AMARAL et al. (1991) e ALBUQUERQUE et al. (1993). O SEXO demonstrou ter efeito significativo sobre o P205 $(\mathrm{P}<0,05)$, com médias superiores para machos, e também sobre a EI, com valores maiores para vacas cujo terneiro era macho $(\mathrm{P}<0,05)$. ALENCAR (1987) também relata efeito do sexo do terneiro sobre o peso aos 240 dias, com machos mais pesados que fêmeas (médias de 197,6 $\pm 1,7$ e 188,5 $\pm 1,8 \mathrm{~kg}$ respectivamente). ALBUQUERQUE et al. (1993) também encontraram resultados semelhantes, trabalhando, entretanto, com peso ajustado para 205 dias. As médias de PN, P205 e EI de acordo com o SEXO podem ser observadas na tabela 3 .

A $\mathrm{PL}_{\mathrm{P} 84}$ e a $\mathrm{PL}_{\mathrm{P} 147}$ tiveram efeito significativo $(\mathrm{P}<0,05)$ sobre o $\mathrm{P} 205$, assim como a $\mathrm{PL}_{\mathrm{P} 210}(\mathrm{P}<0,07)$. Esses resultados estão de acordo com os encontrados por ALENCAR (1987), em que o efeito linear da produção de leite total foi significativo $(\mathrm{P}<0,01)$ sobre o peso do terneiro aos 210 e 240 dias de idade, sendo que $29 \%$ da variação explicada pelo modelo estatístico foi devido à produção de leite total. A eficiência individual das vacas (EI) foi influenciada somente pela $\mathrm{PL}_{\mathrm{P} 84}$ e $\mathrm{PL}_{\mathrm{P} 147}(\mathrm{P}<0,05)$. Os resultados estão de acordo com o esperado, uma vez que, sendo os terneiros dependentes do leite materno na fase

Tabela 3 - Médias de peso ao nascer (PN), peso à desmama ajustado para 205 dias (P205) e eficiência individual das vacas (EI) de acordo com o sexo (SEXO) do terneiro.

\begin{tabular}{lcc}
\hline \multirow{2}{*}{ Variáveis } & \multicolumn{2}{c}{ SEXO } \\
\cline { 2 - 3 } & M (Machos) & F (Fêmeas) \\
\hline PN & $33,26 \pm 1,25 \mathrm{a}$ & $29,32 \pm 0,79 \mathrm{~b}$ \\
P205 & $151,68 \pm 6,41 \mathrm{a}$ & $146,27 \pm 2,73 \mathrm{~b}$ \\
EI & $45,68 \pm 2,75 \mathrm{a}$ & $41,68 \pm 0,94 \mathrm{~b}$ \\
\hline
\end{tabular}

Médias na mesma linha seguidas de letras minúsculas diferentes, diferem significativamente $(\mathrm{P}<0,05)$. inicial da vida, os ganhos nesse período devem-se principalmente ao leite consumido. Com o aumento da idade, os ganhos passam a depender mais da forragem ingerida que do leite consumido, sendo o efeito do leite materno diluído com o tempo (ALENCAR, 1987; ROVIRA, 1996; FRANZO, 1997; JARDIM \& PIMENTEL, 1998).

\section{CONCLUSÕES}

O genótipo do terneiro não demonstrou influência sobre o peso à desmama e sobre a eficiência individual da vaca à desmama, provavelmente em função da limitação nutricional imposta pela condições do estudo e por serem as mães novilhas primíparas, com produção de leite limitada. Isso evidencia que nessas condições, não são verificadas as vantagens e efeitos da heterose sobre as características estudadas. Por outro lado, a época de nascimento precoce demonstrou efeito positivo sobre o peso à desmama $\mathrm{e}$ eficiência da vaca, sendo desta forma, uma importante alternativa de manejo a ser considerada.

\section{REFERÊNCIAS BIBLIOGRÁFICAS}

ALBUQUERQUE, L.G. et al. Produção de leite e desempenho do bezerro na fase de aleitamento em três raças de bovinos de corte. Rev Soc Bras Zoot, v.22, n.5, p.745-754, 1993.

ALENCAR, M.M. Efeitos da produção de leite sobre o desenvolvimento de bezerros Canchim. Rev Soc Bras Zoot, v.16, n.1, p.1-13, 1987.

ALENCAR, M.M.; JUNQUEIRA FILHO, A.A.; PARANHUS, N.E. Produção de leite de vacas da raça Canchim. Rev Soc Bras Zoot, v.14, n.3, p.358-366, 1985.

ALENCAR, M.M. et al. Produção de leite da vaca e desenvolvimento do bezerro em gado de corte. Rev Soc Bras Zoot, v.25, n.1, p. 92-101, 1996.

AMARAL, M.O.; QUEIROZ, S.A.; ALBUQUERQUE, L.G. Efeito da endogamia sobre o peso ao nascer e à desmama de bezerros da raça Caracu. In: REUNIÃO DA SOCIEDADE BRASILEIRA DE ZOOTECNIA, 28., 1991, João Pessoa, PB. Anais... João Pessoa : Sociedade Brasileira de Zootecnia, 1991. p. 540 .

ANC - HERD BOOK COLLARES. Normas e bases para programas uniformes de melhoramento de gado de corte. Projeto de melhoramento de bovinos de corte das raças européias. Pelotas : Grafosul, 1976. 79p. (Boletim n.4).

BARCELLOS, J.O.J.; LOBATO, J.F.P. Efeitos da época no nascimento de bezerros Hereford e suas cruzas. I. Peso ao nascer e ganho médio diário pré-desmama. Rev Soc Bras Zoot, v.21, n.1, p.137-149, 1992a.

BARCELLOS, J.O.J.; LOBATO, J.F.P. Efeitos da época no nascimento de bezerros Hereford e suas cruzas. II. Pesos ao 
desmame, ano e sobreano. Rev Soc Bras Zoot, v.21, n.1, p. $150-157,1992 b$

BOGGS, D.L. et al. Effects of milk and forage intake on calf performance. J Anim Sci, v.51, p.550, 1980.

CARDELlinO, R.; ROVIRA, J. Mejoramiento genetico animal. Montevideo : Hemisferio Sur, 1987. 253p.

FRANZO, V. Produção de leite em vacas primíparas de corte e sua relação com o desenvolvimento dos terneiros. 1997. 102f. Dissertação (Mestrado em Zootecnia) - Faculdade de Agronomia Eliseu Maciel, Universidade Federal de Pelotas.

GREEN, R.D. et al.Output/Input difference's among nonpregnant, lactating Bos indicus - Bos taurus and Bos taurus - Bos taurus F cross cows. J Anim Sci, v.69, n.8, p.3156$3166,1991$.

JARDIM, P.O.C.; PIMENTEL, M.A. Bovinos de corte. Pelotas : UFPel, 1998. 185p.

KOGER, M.; CUNHA, T.J.; WARNICK, A.C. Cruzamientos en ganado vacuno de carne. Montevideo : Hemisferio Sur, 1976. 559p

LOBATO, J.F.P. Bovinos de corte - Seleção e sistemas de acasalamento. Porto Alegre: Adubos Trevo, 1984. 20p.
LOBATO, J.F.P.; BARCELlos, J.O.J.; KESSLER, A.M. Produção de bovinos de corte. Porto Alegre : EDIPUCRS, 1999. $346 \mathrm{p}$

MACEDO, W. Levantamento de reconhecimento dos solos do município de Bagé, RS. Bagé : EMBRAPA, 1984. 69p.

MALLINCKRODT, C.H. et al. Relationship of maternal milk expected progeny differences to actual milk yield and calf weaning weight. J Anim Sci, v.71, p.355-362, 1993.

NEWMAN, S.; DELAND, M.P. Lifetime productivity of crossbred cows. 2 Age and weight at first oestrus, calf birth weight, assisted calvings, calving interval and reproduction rate. Austr J Exp Agric, Port Melbourne, v.31, n.3, p.293$300,1991$.

RIBEIRO, A.M.L.; LOBATO, J.F.P. Produtividade e eficiência reprodutiva de três grupos raciais de novilhas de corte. I. Desempenho reprodutivo. Rev Soc Bras Zoot, v.17, p.498- 507, 1988.

ROVIRA, J. Reproduccion y manejo de los rodeos de cria. Montevideo : Hemisferio Sur, 1974. 296p.

ROVIRA, J. Manejo nutritivo de los rodeos de cría en pastoreo. Montevideo : Hemisferio Sur, 1996. 288p.

SAS. Statistical Analysis System, Version 7 (TS P1) for WIN-95 platform. Cary, NC : SAS Institute, 2000. 